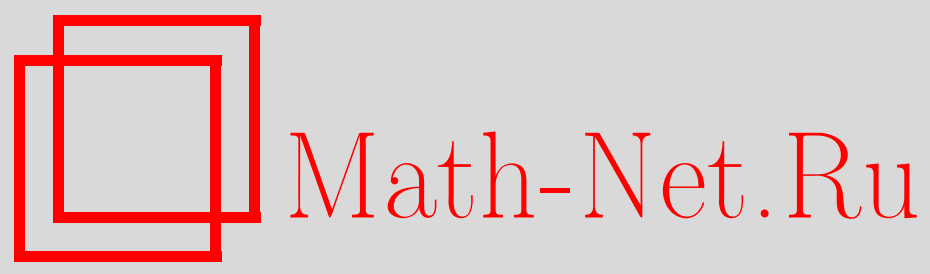

Н. М. Тимофеев, М. Б. Хрипунова, О функции концентрации аддитивной функции с немультипликативным весом, Матем. заметки, 2004, том 75, выпуск 6, 877-894

DOI: https://doi.org/10.4213/mzm78

Использование Общероссийского математического портала Math-Net.Ru подразумевает, что вы прочитали и согласны с пользовательским соглашением http://www.mathnet.ru/rus/agreement

Параметры загрузки:

IP : 35.173 .137 .237

26 апреля 2023 г., 14:55:39 


\section{О ФУНКЦИИ КОНЦЕНТРАЦИИ АДДИТИВНОЙ ФУНКЦИИ С НЕМУЛЬТИПЛИКАТИВНЫМ ВЕСОМ}

\section{Н.М. Тимофеев, М.Б. Хрипунова}

Пусть $g(n)$ - аддитивная функция, принимающая вещественные значения, $\tau(n)$ число делителей $n$. В работе доказано, что существует постоянная $C$ такая, что

$$
\sup _{a} \sum_{\substack{n<N \\ g(n) \in[a, a+1)}} \tau(N-n) \leqslant C \frac{N \log N}{\sqrt{W(N)}}
$$

где

$$
W(N)=4+\min _{\lambda}\left(\lambda^{2}+\sum_{p<N} \frac{1}{p} \min \left(1,(g(p)-\lambda \log p)^{2}\right)\right) .
$$

Из этого результата, в частности, следует, что

$$
\sup _{a}|\{m, n: m n<N, g(N-m n)=a\}| \ll N \log N\left(\sum_{p<N, g(p) \neq 0}(1 / p)\right)^{-1 / 2} .
$$

Подразумеваемая константа является абсолютной.

Библиографоия: 12 названий.

1. Введение и основные результаты. Пусть $g(n)$ - аддитивная функция, принимающая вещественные значения. Напомним, что функция $g$ называется аддитивной, если $g(n \cdot m)=g(n)+g(m)$ для всех натуральных, взаимно простых чисел $n, m$ и $g(0)=0$.

В 1980 году Ружа [1] доказал следуюший результат.

ТЕОремА А. Существует абсолютная постоянная такая, что для любой аддитивной функиии справедливо неравенство

$$
\frac{1}{x} \sup _{a}|\{n: n \leqslant x, a \leqslant g(n)<a+1\}| \leqslant \frac{c}{\sqrt{W(x)}}
$$

$2 \partial e$

$$
W(x)=4+\min _{\lambda}\left(\lambda^{2}+\sum_{p \leqslant x} \frac{1}{p} \min \left(1,(g(p)-\lambda \log p)^{2}\right)\right) .
$$

Это неравенство оказалось очень полезньп для изучения других интегральных и локальных характеристик аддитивных функций. Например, из него легко вытекает следуюший результат Халоса (см. [2]).

Работа выполнена при финансовой поддержке Российского фонда фундаментальных исследований, грант № 99-01-00070. 
ТЕоремА В. Существует абсолютная постоянная такая, что для любой аддитивной функиии

$2 \partial e$

$$
\sup _{a} \frac{1}{x}|\{n: n \leqslant x, g(n)=a\}| \leqslant \frac{C}{E(x)}
$$

$$
E(x)=4+\sum_{\substack{p \leqslant x \\ g(p) \neq 0}} \frac{1}{p}
$$

В [3] Тимофеев доказал следующий аналог неравенства Ружи для множества сдвинутых простых чисел.

Теорема С. Пусть $b \neq 0$ - челое, фиксированное число, $p$ - простое число. Существует постоянная $C$, зависящая только от $b$, такая, что

$$
\sup _{a} \frac{1}{\pi(x)}|\{p: p+b \leqslant x, a \leqslant g(p+b)<a+1\}| \leqslant \frac{C \log ^{2} W(x)}{\sqrt{W(x)}} .
$$

Этот результат позволил перенести на множество сдвинутьх простых чисел аналог неравенства типа Халоса и теоремы о сходимости последовательности функций распределения

$$
\frac{1}{\pi(x)}|\{p: p+b \leqslant x, g(p+b)-A(x)<u B(x)\}|
$$

к предельной функции $F(u)$ во всех точках непрерьвности последней.

В 1994 году Эллиот [4] показал, что неравенство (1) справедливо без множителя $\log ^{2} W(x)$. В таком виде неравенство (1), как и неравенство Ружи, точны по порядку. Это означает, что существуют аддитивные функции, для которых левая часть соотношения (1) не меньше, чем $C_{1}(W(x))^{-1 / 2}$, где $C_{1}>0$. В работе [4] Эллиот доказал также следующий результат.

ТЕорема D. Существует абсолютная постоянная такая, что для всех аддитивных функций при $N \geqslant 3$ справедливо неравенство

$$
\frac{1}{\pi(N)} \sup _{a}|\{p: p<N, a \leqslant g(N-p)<a+1\}| \leqslant \frac{C}{\sqrt{Y(N)}}
$$

$2 \partial e$

$$
Y(N)=4+\min _{\lambda}\left(\lambda^{2}+\sum_{\substack{p<N \\(p, n)=1}} \min \left(1,|g(p)-\lambda \log p|^{2}\right)\right) .
$$

Используя метод работы [1], идеи и результаты работ [3], [4], здесь мы доказали следуюшие теоремы.

Теорема 1. Существует абсолютная постоянная $C$ такая, что для любой аддитивной функиии при $N \geqslant 2$ справедливо неравенство

$$
\sup _{a} \sum_{\substack{n<N \\ a \leqslant g(n)<a+1}} \tau(N-n) \leqslant \frac{C N \log N}{\sqrt{W(N)}}
$$

әде $\tau(n)$ - число делителей $n$.

В теореме 1 равномерно относительно $а$ оценено число представлений $N$ в виде суммы $n+k l$, где $n, k, l$ - натуральные числа, причем $a \leqslant g(n)<a+1$.

Отсюда, действуя так же, как и в [1], можно вывести следующий результат. 
СлЕДСтвиЕ 1. Существует абсолютная постоянная такая, что для любой аддитивной функиии при $N \geqslant 2$ справедливо неравенство

$$
\sup _{a} \sum_{\substack{n<N \\ g(n)=a}} \tau(N-n) \leqslant \frac{C N \log N}{\sqrt{E(N)}}
$$

əəe

$$
E(N)=4+\sum_{\substack{p<N \\ g(p) \neq 0}} \frac{1}{p}
$$

ДоКАЗАТЕЛЬСТво. Положим при $N \geqslant 2$

$$
\frac{1}{N \log N} \sum_{\substack{n<N \\ g(n)=a}} \tau(N-n)=A(N)
$$

Для тех $N$, для которых $A(N)=0$, неравенство (2) справедливо, поэтому можно считать, что $A(N) \neq 0$.

При любом $h>0$ имеем

$$
\sum_{\substack{n<N \\ a / h-1 / 2 \leqslant g(n) / h<a / h+1 / 2}} \tau(N-n) \geqslant A(N) N \log N,
$$

и тогда из теоремы 1 следует существование $\lambda(h)$ такого, что

$$
W(n)=4+\lambda^{2}(h)+\sum_{p<N} \frac{1}{p} \min \left(1,\left(\frac{g(n)}{h}-\lambda(h) \log p\right)^{2}\right) \leqslant C^{2} A^{-2}(N) .
$$

Отсюда вытекает, что $|\lambda(h)| \leqslant C A^{-1}(N)$. Следовательно, для тех $p$, для которых $g(p) \neq 0$, имеем

$$
\lim _{h \rightarrow 0}\left(\frac{g(p)}{h}-\lambda(h) \log p\right)^{2}=\infty .
$$

Переходя к пределу при $h \rightarrow 0$ в неравенстве

$$
4+\sum_{p<N} \frac{1}{p} \min \left(1,\left(\frac{g(p)}{h}-\lambda(h) \log p\right)^{2}\right) \leqslant C^{2} A^{-2}(N),
$$

получим $E(N) \leqslant C^{2} A^{-2}(N)$, т.е. неравенство (2).

В следствии 1 оценено число представлений $N$ в виде суммы $n+k l$, где $n, k, l$ - натуральные числа и $g(n)=a$. Причем полученная оценка равномерна по $a$ и $g$.

Мы приведем доказательство теоремы 1 ниже. Несколько проще, чем теорема 1 , доказывается 
Теорема 2. Пусть $b \neq 0$ - иелое число. Существует постоянная $C(b)$, зависящая только от $b$, такая, что для всех аддитивных функиий при $x \geqslant 3$ справедливо неравенство

$$
\sup _{a} \sum_{\substack{0<n+b \leqslant x \\ a \leqslant g(n)<a+1}} \tau(n+b) \leqslant \frac{C(b) x \log x}{\sqrt{W(x)}} .
$$

Отсюда, как и в предыдущем случае, вытекает

СледСТВИЕ 2. Существует постоянная $C$, зависящая только от $b$, такая, что для всех аддитивных функиий выполнено неравенство

$$
\sup _{\substack{a \\ 0}} \sum_{\substack{0, n+b \leqslant x \\ g(n)=a}} \tau(n+b) \leqslant \frac{C x \log x}{E(x)}
$$

Убедимся, что полученные результаты точны по порядку. Суммы, стоящие в правых частях неравенств (2) и $(3)$, в случае $g(n)=\Omega(n)$, где $\Omega(n)$ - число простых делителей $n$ с учетом их кратности, изучались соответственно в работах [5] и [6]. В работе [5] (см. теорему 3) было показано, что при $k \leqslant(2-\varepsilon) \log \log x$

$$
\begin{aligned}
\sum_{\substack{n \leqslant x \\
\Omega(n)=k}} \tau(n-1)= & x \prod_{p}\left(1+\frac{1}{p(p-1)}\right)\left(1-\frac{k-1}{\left(p^{2}-p+1\right) \log \log x}\right) \\
& \times F\left(\frac{k}{\log \log x}\right) \frac{(\log \log x)^{k-1}}{(k-1) !}\left(1+O_{\varepsilon}\left(\frac{1}{\sqrt{\log \log x}}\right)\right),
\end{aligned}
$$

где

$$
F(z)=\frac{1}{\Gamma(z+1)} \prod_{p}\left(1-\frac{1}{p}\right)^{z}\left(1-\frac{z}{p}\right)^{-1} .
$$

Выберем $k=\log \log x+O(1)$. Тогда, применив формулу Стирлинга, получим, что левая часть соотношения (4) будет

$$
\geqslant C_{1} \frac{x \log x}{\sqrt{\log \log x}}
$$

где $C_{1}>0$. Таким образом, мы доказали, что неравенство (3) точно по порядку. Используя теорему работы [6], точно так же можно показать, что и неравенство (2) точно по порядку.

2. Вспомогательные результаты. Будем далее обозначать через $p(n)$ и $q(n)$ наибольший и наименьший простой делитель $n$ соответственно, $p(1)=q(1)=1$.

ЛЕмма 1. Пусть $2 \leqslant r \leqslant x$. Тогда

$$
|\{n: n \leqslant x, p(n) \leqslant r\}| \ll x \exp \left(-\frac{\log x}{2 \log r}\right) .
$$


ДокАЗАТЕЛЬСТво этой леммы приведено, например, в [7, раздел 3.5, теорема 1].

Нам потребуется результат Халоса (см. [1], [2]). Мы приведем его в форме, доказанной в книге [7, гл. 4 , следствие 6.3$]$.

Лемма 2. Существует абсолютная постоянная с такая, что для любой мультипликативной функиии $f,|f(n)| \leqslant 1$, справедливо неравенство

$$
\left|\sum_{n \leqslant x} f(n)\right| \leqslant c x\left(\exp \left(-\frac{1}{2} \min _{|t| \leqslant T} \sum_{p \leqslant x} \frac{1-\operatorname{Re} f(p) p^{-i t}}{p}\right)+\frac{1}{\sqrt{T}}\right) .
$$

Лемма 3. Пусть $|f(p)| \leqslant 1$ для всех простых иисел $p$. Предположим, что найдется примитивныи иарактер модуля $\delta, \delta \leqslant \log ^{2} N$, и вещественное число $\lambda_{0}$, $\left|\lambda_{0}\right| \leqslant \log ^{2} N$, такие, что

$$
\sum_{p \leqslant N} \frac{1}{p}\left(1-\operatorname{Re} f(p) p^{-i \lambda_{0}} \chi_{\delta}(p)\right) \leqslant \frac{1}{100} \log \log N .
$$

Тогда для всех характеров Дирихле $\chi_{d} c d \leqslant \log ^{2} N$, не порожденных $\chi_{\delta}$, при $|\lambda| \leqslant \log ^{2} N$ справедливо неравенство

$$
\sum_{p \leqslant N} \frac{1}{p}\left(1-\operatorname{Re} f(p) p^{-i \lambda} \chi_{d}(p)\right) \geqslant \frac{1}{2} \log \log N+O(1) .
$$

ДокаЗАТЕЛЬСТво. Имеем

$$
\begin{aligned}
\sum_{p \leqslant N} \frac{1-\operatorname{Re} f(p) p^{-i \lambda} \chi_{d}(p)}{p} \geqslant & \sum_{\substack{p \leqslant N \\
p \nmid \delta}} \frac{1-\operatorname{Re} p^{-i\left(\lambda-\lambda_{0}\right)} \chi_{d}(p) \overline{\chi_{\delta}(p)}}{p} \\
& -\sum_{p \leqslant N} \frac{\left|1-f(p) p^{-i \lambda_{0}} \chi_{\delta}(p)\right|}{p} .
\end{aligned}
$$

Учитывая, что

$$
\left|1-f(p) p^{-i \lambda_{0}} \chi_{\delta}(p)\right| \leqslant \sqrt{2\left(1-\operatorname{Re} f(p) p^{-i \lambda_{0}} \chi_{\delta}(p)\right)},
$$

применив неравенство Коши, получим, что вторая сумма

$$
\leqslant\left(\sum_{p \leqslant N} \frac{1}{p} \cdot 2 \sum_{p \leqslant N} \frac{1-\operatorname{Re} f(p) p^{-i \lambda_{0}} \chi_{\delta}(p)}{p}\right) \leqslant \frac{1}{5} \log \log N+O(1) .
$$

Первая сумма при $\sigma_{N}=1+1 / \log N$ равна

$$
\begin{aligned}
\sum_{p} & \frac{1}{p^{\sigma_{N}}}-\operatorname{Re} \sum_{p} \frac{\chi_{d}(p) \overline{\chi_{\delta}(p)}}{p^{\sigma_{N}+i\left(\lambda-\lambda_{0}\right)}}+O\left(\sum_{p \mid \delta} \frac{1}{p}\right)+O(1) \\
\quad & =\log \zeta\left(\sigma_{N}\right)-\log \left|L\left(\sigma_{N}+i\left(\lambda-\lambda_{0}\right), \chi_{d} \overline{\chi \delta}\right)\right|+O(\log \log \log N) .
\end{aligned}
$$


Так как $\chi_{d} \overline{\chi \delta}-$ неглавный характер, используя хорошо известные результаты

$$
\begin{gathered}
\log \zeta\left(\sigma_{N}\right)=\log \log N+O(1), \\
\mid L\left(\sigma_{N}+i\left(\lambda-\lambda_{0}, \chi_{d} \overline{\chi \delta}\right) \mid \ll \log \left(d \delta\left(\left|\lambda-\lambda_{0}\right|+2\right)\right),\right.
\end{gathered}
$$

получим утверждение леммы 3 .

При доказательстве леммы 4 (см. далее) мы будем применять метод Халоса [8], в котором используются следующие оценки. Пусть $f$-мультипликативная функция, $|f(n)| \leqslant 1$,

$$
F(s)=\sum_{n=1}^{\infty} \frac{f(n)}{n^{s}} .
$$

Тогда при $s=\sigma+i t, \sigma=1+(\log x)^{-1}, x \geqslant 2$, имеем (см., например, [9, с. 233-235])

$$
\begin{gathered}
\left|\frac{F(s)}{\zeta(s)}\right| \ll \exp \left(\sum_{p \leqslant x} \frac{|f(p)-1|}{p}\right), \\
\int_{-\infty}^{+\infty}\left|\frac{F(s)}{s}\right|^{2} d t \ll \frac{1}{\sigma-1}, \\
\int_{-\infty}^{+\infty}\left|\frac{1}{s} \frac{F^{\prime}}{F}(s)\right|^{2} d t \ll \frac{1}{\sigma-1} .
\end{gathered}
$$

Лемма 4. Существует абсолютная постоянная $c>0$ такая, что для любой мультипликативной функиии $f,|f(n)| \leqslant 1$, выполняется неравенство

$$
\begin{aligned}
& \left|\sum_{\substack{n \leqslant x \\
(n, N)=1}} f(n)-\frac{\varphi(N)}{N} \sum_{n \leqslant x} f(n)\right| \\
& \quad \leqslant c x\left(\sum_{d \mid N} \frac{1+\log d}{d}\right)^{1 / 2}(\log x)^{-1 / 4} \exp \left(\frac{1}{2} \sum_{p \leqslant x} \frac{1}{p}|f(p)-1|\right) .
\end{aligned}
$$

ДокАЗАТЕЛЬСТво. При любом $y \in[2,2 x]$ и $\sigma=1+(\log x)^{-1}$ имеем

$$
\sum_{n \leqslant y} f(n) \log n \log \frac{y}{n}=-\frac{1}{2 \pi i} \int_{\operatorname{Re} s=\sigma} \frac{y^{s}}{s^{2}} F^{\prime}(s) d s+O(1) .
$$

Отсюда следует, что

$$
\begin{aligned}
& \left|\sum_{\substack{n \leqslant y \\
(n, N)=1}} f(n) \log n \log \frac{y}{n}-\frac{\varphi(N)}{N} \sum_{n \leqslant y} f(n) \log n \log \frac{y}{n}\right| \\
& \quad \leqslant \frac{1}{2 \pi} \int_{-\infty}^{+\infty} \frac{y^{\sigma}}{|s|^{2}}\left|\left(\sum_{d \mid N} \frac{\mu(d)}{d^{s}} \cdot \sum_{n} \frac{f(n)}{n^{s}}\right)^{\prime}-\frac{\varphi(N)}{N}\left(\sum_{n} \frac{f(n)}{n^{s}}\right)^{\prime}\right| d t \\
& \quad \leqslant \frac{1}{2 \pi} \int_{-\infty}^{+\infty} \frac{y^{\sigma}}{|s|^{2}}\left|\sum_{d \mid N} \mu(d)\left(\frac{1}{d^{s}}-\frac{1}{d}\right)\right| \cdot\left|F^{\prime}(s)\right| d t+\frac{1}{2 \pi} \sum_{d \mid N} \frac{\log d}{d} \int_{-\infty}^{+\infty} \frac{y^{\sigma}}{|s|^{2}}|F(s)| d t .
\end{aligned}
$$


Первый интеграл разобьем на две части: $|t| \leqslant 1$ и $|t|>1$. При $|t| \leqslant 1$ воспользуемся оценкой

$$
\left|\sum_{d \mid N} \mu(d)\left(\frac{1}{d^{s}}-\frac{1}{d}\right)\right| \ll|s-1| \sum_{d \mid N} \frac{\log d}{d},
$$

а при $|t|>1$ левая часть будет

$$
\leqslant 2 \sum_{d \mid N} \frac{1}{d}
$$

Следовательно, первый интеграл сверху оценивается так:

$$
\begin{aligned}
& \ll x \sum_{d \mid N} \frac{1+\log d}{d} \\
& \quad \times\left(\int_{-1}^{1}\left|\frac{F^{\prime}}{F}(s)\right| \cdot\left|\frac{F(s)}{\zeta(s)}\right| \cdot|\zeta(s)(s-1)| \frac{d t}{|s|^{2}}+\int_{|t|>1}\left|\frac{F^{\prime}}{F}(s)\right| \cdot\left|\frac{F(s)}{\zeta(s)}\right| \cdot|\zeta(s)| \frac{d t}{|s|^{2}}\right) .
\end{aligned}
$$

Воспользуемся (5) и известными оценками:

$$
\begin{gathered}
|\zeta(s)(s-1)|=O(1) \quad \text { при } \sigma>1, \quad|t| \leqslant 1, \\
\zeta(s)=O(\log 2|t|) \quad \text { при } \sigma>1,|t| \geqslant 1 .
\end{gathered}
$$

Применив неравенство Шварца и (7), получим, что оцениваемый интеграл

$$
\ll x(\log x)^{1 / 2} \sum_{d \mid N} \frac{1+\log d}{d} \exp \left(\sum_{p \leqslant x} \frac{1}{p}|f(p)-1|\right) .
$$

Для второго интеграла в правой части (8) после применения неравенства Шварца и (6) получим такую же оценку. Таким образом, имеем

$$
\sum_{\substack{n \leqslant y \\(n, N)=1}} f(n) \log n \log \frac{y}{n}=\frac{\varphi(N)}{N} \sum_{n \leqslant y} f(n) \log n \log \frac{y}{n}+O(x R(x)),
$$

где

$$
R(x)=\sqrt{\log x} \sum_{d \mid N} \frac{1}{d}(1+\log d) \exp \left(\sum_{p \leqslant x} \frac{1}{p}|f(p)-1|\right) .
$$

Возьмем $y=x$ и $y=x(1-\varepsilon), \varepsilon=\sqrt{R(x) / \log x}$ и вычтем друг из друга вновь полученные равенства. После деления на $\varepsilon$ находим

$$
\sum_{\substack{n \leqslant x \\(n, N)=1}} f(n) \log n=\frac{\varphi(N)}{N} \sum_{n \leqslant x} f(n) \log n+O(x \sqrt{R(x) \log x}) .
$$

Учитьвая, что

$$
\sum_{n \leqslant x} f(n) \log n=\log x \sum_{n \leqslant x} f(n)+O(x),
$$

отсюда получим утверждение леммы 4.

Следующая лемма доказана в работе [10]. 
Лемма 5. Если $Q \leqslant x, f(n) \geqslant 0, m o$

$$
\sum_{k \leqslant Q} \max _{(a, k)=1}\left(\sum_{\substack{n \leqslant x \\ n \equiv a(\bmod k)}} f(n)+\frac{1}{\varphi(k)} \sum_{n \leqslant x} f(n)\right) \ll\left(\sum_{n \leqslant x} f^{2}(n)\right)^{1 / 2}\left(Q+\sqrt{x} \log ^{3 / 2} x\right) .
$$

Лемма 6. Пусть $\left\{a_{n}\right\},\left\{b_{m}\right\}$ - последовательности комплексных чисел такие, что $a_{n}=0$ при $n \leqslant z_{1}, b_{m}=0$ при $m \leqslant z_{2}, d_{1}, d_{2}$ - натуральные числа, причем $p\left(d_{1}\right) \leqslant z, q\left(d_{2}\right)>z$. Тогда

$$
\begin{aligned}
\Delta(Q):= & \sum_{d=d_{1} d_{2} \leqslant Q} \max _{(a, d)=1}\left|\sum_{\substack{n m \leqslant x \\
n m \equiv a(\bmod d)}} a_{n} b_{m}-\frac{1}{\varphi\left(d_{2}\right)} \sum_{\substack{n m \leqslant x \\
\left(n m, d_{2}\right)=1 \\
n m \equiv a\left(\bmod d_{1}\right)}} a_{n} b_{m}\right| \\
\ll & \left(\frac{1}{\log ^{A} x}\left(\sum_{k \leqslant 2 x}\left(\sum_{n m=k}\left|a_{n} b_{m}\right|\right)^{2}\right)^{1 / 2}\right. \\
& \left.+\left(\sum_{|k-x| \leqslant x / \log A}\left(\sum_{n m=k}\left|a_{n} b_{m}\right|^{2}\right)\right)^{1 / 2}\right)\left(Q+\sqrt{x} \log ^{3 / 2} x\right) \\
& +(\log \log x) \max _{y \leqslant x}\left(\frac{1}{x} \sum_{n \leqslant y}\left|a_{n}\right|^{2} \sum_{m \leqslant x / y}\left|b_{m}\right|^{2}\right)^{1 / 2} \\
& \times\left(Q \sqrt{x} \log \frac{x}{z_{1} z_{2}}+\left(\frac{x}{\sqrt{z_{2}}}+\frac{x}{\sqrt{z_{1}}}\right) \log ^{2} Q+\frac{x}{z}(\log Q) \log \frac{x}{z_{1} z_{2}}\right)
\end{aligned}
$$

где $A$ - любая положительная постоянная.

ДокАзАТЕЛьство. Разобьем промежуток изменения $n$, т.е. $\left(z_{1}, x / z_{2}\right)$, на промежутки вида

$$
\left(U_{j}, U_{j+1}\right], \quad \text { где } U_{j}=2^{j} z_{1}, \quad 0 \leqslant j \leqslant \log _{2} \frac{x}{z_{2} z_{1}}=r .
$$

Последний промежуток может быть неполньм. С помощью разрьвного множителя

$$
\int_{-\infty}^{+\infty} n^{i \alpha} \frac{\sin \alpha \log ([x]+1 / 2)}{\alpha} d \alpha=\left\{\begin{array}{l}
\pi, \text { если } n \leqslant x, \\
0, \text { если } n>x
\end{array}\right.
$$

сделаем суммирование по $n$ и $m$ независимым друг от друга. В результате получим

$$
\begin{aligned}
& \Delta(Q) \ll \sum_{d=d_{1} d_{2} \leqslant Q} \sum_{0 \leqslant j \leqslant r} \max _{(a, d)=1} \mid \int_{-\infty}^{+\infty} \frac{\sin \alpha \log ([x]+1 / 2)}{\alpha} \\
& \times \sum_{\substack{U_{j}<n \leqslant U_{j+1} \\
(n, d)=1}} a_{n} n^{i \alpha}\left(\sum_{\substack{z_{2}<m \leqslant x / U_{j} \\
n m \equiv a(\bmod d)}} b_{m} m^{i \alpha}-\frac{1}{\varphi\left(d_{2}\right)} \sum_{\substack{z_{2}<m \leqslant x / U_{j} \\
n m \equiv a\left(\bmod d_{1}\right) \\
\left(m, d_{2}\right)=1}} b_{m} m^{i \alpha}\right) \mid d \alpha .
\end{aligned}
$$

Промежуток интегрирования разобьем на три части:

$$
|\alpha| \geqslant x^{5}, \quad(\log x)^{2 A} \leqslant|\alpha| \leqslant x^{5}, \quad|\alpha| \leqslant \log ^{2 A} x,
$$


где $A \geqslant 4$. Если $n m$ не входит в

$$
I=\left[x-\frac{x}{\log ^{A} x}, x+\frac{x}{\log ^{A} x}\right]
$$

то в первом и втором интегралах воспользуемся оценкой

$$
\left|\int_{|\alpha| \geqslant t} n^{i \alpha} \frac{\sin \alpha \log ([x]+1 / 2)}{\alpha} d \alpha\right| \ll \frac{1}{|t| \log ((2[x]+1) /(2 n))} \ll \frac{\log ^{A} x}{|t|} .
$$

Если $n m \in I$, то эти интегралы оценим как $O(\log x)$. После применения леммы 5 получим, что эти части дадут вклад в $\Delta(Q)$, оцениваемый через

$$
\begin{aligned}
& \ll\left(\frac{1}{\log ^{A} x}\left(\sum_{k \leqslant 2 x}\left(\sum_{n m=k}\left|a_{n} b_{m}\right|\right)^{2}\right)^{1 / 2}\right. \\
& \left.+\left(\sum_{x-x / \log ^{A} x \leqslant k \leqslant x+x / \log ^{A} x}\left(\sum_{n m=k}\left|a_{n} b_{m}\right|\right)^{2}\right)^{1 / 2}\right)\left(Q+\sqrt{x}(\log x)^{3 / 2}\right) .
\end{aligned}
$$

Оставшаяся часть суммы $\Delta(Q)$ (обозначим ее через $\left.\Delta_{1}(Q)\right)$ будет оцениваться так:

$$
\Delta_{1}(Q) \ll \log \log x \max _{\alpha} \sum_{\substack{d_{1} d_{2} \leqslant Q \\ d_{2}>z}} \frac{1}{\varphi\left(d_{1} d_{2}\right)} \sum_{\substack{\chi_{d_{1}} \chi_{d_{2}} \\ \chi_{d_{2}} \neq \chi_{0}}} \sum_{j=0}^{r} \mid S_{j}\left(\chi _ { d _ { 1 } d _ { 2 } } | \cdot | F _ { j } \left(\chi_{d_{1} d_{2}} \mid\right.\right.
$$

где

$$
S_{j}\left(\chi_{d}\right)=\sum_{U_{j}<n \leqslant U_{j+1}} \chi_{d}(n) a_{n} n^{i \alpha}, \quad F_{j}\left(\chi_{d}\right)=\sum_{m \leqslant x / U_{j}} \chi_{d}(m) a_{m} m^{i \alpha} .
$$

Перейдем к примитивным характерам. Так как $\chi_{d_{2}} \neq \chi_{0}$ и все простые делители $d_{2}$ больше $z$, то модуль примитивного характера, порождающего $\chi_{d_{1}} \chi_{d_{2}}$, будет больше $z$. Таким образом, получим

$$
\Delta_{1}(Q) \ll \log \log x \max _{\alpha} \sum_{0 \leqslant j \leqslant r} \sum_{1 \leqslant d \leqslant Q} \frac{1}{\varphi(d)} \sum_{z<k \leqslant Q / d} \frac{1}{\varphi(k)} \sum_{\chi_{k}^{*}}\left|S_{j, d}\left(\chi_{k}^{*}\right)\right| \cdot\left|F_{j, d}\left(\chi_{k}^{*}\right)\right|
$$

Здесь $\chi_{k}^{*}$ - примитивный характер модуля $k$, а суммы $S_{j, d}, F_{j, d}$ те же, что и $S_{j}, F_{j}$ по $(n, d)=1$ и $(m, d)=1$.

Разобьем промежуток $(z, Q / d]$ на промежутки вида $\left[2^{t} z, 2^{t+1} z\right]$, где $0 \leqslant t \leqslant T=$ $\log _{2}(Q / d z)$. После применения неравенства Коши получим

$$
\begin{aligned}
\Delta_{1}(Q) \ll & \log \log x \max _{\alpha} \sum_{0 \leqslant j \leqslant r} \sum_{d \leqslant Q} \frac{1}{\varphi(d)} \sum_{0 \leqslant t \leqslant T} \frac{1}{2^{t} z} \\
& \times\left(\sum_{k \leqslant 2^{t+1} z} \frac{k}{\varphi(k)} \sum_{\chi_{k}^{*}}\left|S_{j, d}\left(\chi_{k}^{*}\right)\right|^{2}\right)^{1 / 2}\left(\sum_{k \leqslant 2^{t+1} z} \frac{k}{\varphi(k)} \sum_{\chi_{k}^{*}}\left|F_{j, d}\left(\chi_{k}^{*}\right)\right|^{2}\right)^{1 / 2} .
\end{aligned}
$$


Применив большое решето в форме Галахера (см. [11])

$$
\sum_{k \leqslant Q} \frac{k}{\varphi(k)} \sum_{\chi_{k}^{*}}\left|\sum_{M<n \leqslant M+N} C_{n} \chi_{k}^{*}(n)\right|^{2} \leqslant\left(Q^{2}+\pi N\right) \sum_{M<n \leqslant M+N}\left|C_{n}\right|^{2},
$$

находим

$$
\begin{aligned}
\Delta_{1}(Q) \ll & \log \log x \sum_{0 \leqslant j \leqslant r} \sum_{d \leqslant Q} \frac{1}{\varphi(d)} \sum_{0 \leqslant t \leqslant T} \frac{1}{2^{t} z} \\
& \times\left(\left(\left(2^{t} z\right)^{2}+2^{j} z_{1}\right) \sum_{n \leqslant 2^{j} z_{1}}\left|a_{n}\right|^{2}\left(\left(2^{t} z\right)^{2}+\frac{x}{2^{j} z_{1}}\right) \sum_{m \leqslant x /\left(2^{j} z_{1}\right)}\left|b_{m}\right|^{2}\right)^{1 / 2} \\
\ll & (\log \log x) \max _{y \leqslant x}\left(\frac{1}{x} \sum_{n \leqslant y}\left|a_{n}\right|^{2} \sum_{m \leqslant x / y}\left|b_{m}\right|^{2}\right)^{1 / 2} \\
& \times\left(Q \sqrt{x} \log \frac{x}{z_{1} z_{2}}+\left(\frac{x}{\sqrt{z_{2}}}+\frac{x}{\sqrt{z_{1}}}\right) \log ^{2} Q+\frac{x}{z}(\log Q) \log \log \frac{x}{z_{1} z_{2}}\right) .
\end{aligned}
$$

Учитывая (9), отсюда получим утверждение леммы 6.

3. ДоКАЗАТЕЛЬСТво ТЕОРЕмЫ 1. Мы приведем доказательство наиболее сложного случая, т.е. оценим

$$
X(N):=\sup _{a} \sum_{\substack{n<N \\ g(n) \in[a, a+1)}} \tau(N-n) .
$$

Покажем, что для доказательства теоремы 1 достаточно показать, что

$$
X^{*}(N):=\sup _{a} \sum_{\substack{n<N \\(n, N)=1 \\ g(n) \in[a, a+1)}} \tau(N-n) \ll\left(\frac{\varphi(N)}{N}\right)^{2} \frac{N \log N}{\sqrt{W(N, g)}} .
$$

Напомним, что

$$
W(N, g)=4+\min _{\lambda}\left(\lambda^{2}+\sum_{p<N} \frac{1}{p} \min \left(1,(g(p)-\lambda \log p)^{2}\right)\right) .
$$

В тех местах, где аддитивная функция одна и та же, будем обозначать $W(N, g)$ через $W(N)$.

Действительно,

$$
X(N) \leqslant \sum_{d \mid N} \tau(d) \sup _{a} \sum_{\substack{n<N / d,(n, N / d)=1 \\ g(n d)-g(d) \in[a-g(d), a-g(d)+1)}} \tau\left(\frac{N}{d}-n\right) .
$$

Функция $g_{d}(n)=g(n d)-g(d)$ аддитивная, поэтому, используя $(10)$, имеем

$$
X(N) \ll \sum_{d \mid N} \tau(d)\left(\frac{\varphi(N / d)}{N / d}\right)^{2} \frac{(N / d) \log (N / d)}{\sqrt{W\left(N / d, g_{d}\right)}}
$$


Заметим, что $g(p d)-g(d)=g(p)$, если $p \nmid d$, поэтому при $d \leqslant \sqrt{N}$

$$
W\left(\frac{N}{d}, g_{d}\right)=W(N, g)+\Theta\left(\sum_{\sqrt{N} \leqslant p \leqslant N} \frac{1}{p}+\sum_{p \mid d} \frac{1}{p}\right),
$$

где $|\Theta| \leqslant 1$ и

$$
\frac{1}{\sqrt{W\left(N / d, g_{d}\right)}}-\frac{1}{\sqrt{W(N, g)}} \ll \frac{1}{\sqrt{W(N, g)}}\left(1+\sum_{p \mid d} \frac{1}{p}\right) .
$$

Следовательно, получаем

$$
\begin{aligned}
X(N) \ll & \left(\frac{\varphi(N)}{N}\right)^{2} N \log N \sum_{d \mid N} \frac{\tau(d)}{d}\left(\frac{d}{\varphi(d)}\right)^{2}\left(1+\sum_{p \mid d} \frac{1}{p}\right) \frac{1}{\sqrt{W(N, g)}} \\
& +N \sum_{d \mid N} \frac{\tau(d)}{d} \log d .
\end{aligned}
$$

Отсюда, учитьвая, что

$$
\begin{aligned}
\sum_{d \mid N} & \frac{\tau(d)}{d}\left(\frac{d}{\varphi(d)}\right)^{2}\left(1+\sum_{p \mid d} \frac{1}{p}\right) \\
& \ll\left(1+\sum_{p \mid N} \frac{2}{(p-1)}\right) \prod_{p \mid N}\left(1+\sum_{r=1}^{\infty} \frac{\tau\left(p^{r}\right)}{p^{r}}\left(\frac{p}{p-1}\right)^{2}\right) \ll\left(\frac{N}{\varphi(N)}\right)^{2}
\end{aligned}
$$

и

$$
\sum_{d \mid N} \frac{\tau(d)}{d} \log d \ll \sum_{p^{r} \mid N} \frac{(r+1) \log p^{r}}{p^{r}} \sum_{d \mid N} \frac{\tau(d)}{d} \ll(\log \log N)^{3},
$$

получим неравенство теоремы 1.

Так как $(\varphi(N) / N)^{2} \gg(\log \log N)^{-2}$ и $W(N) \ll \log \log N$, то части суммы $X^{*}(N)$, которые равны $O\left(N(\log N)(\log \log N)^{-3}\right)$, будут удовлетворять неравенству (10). Выделим части, допускающие эту оценку. Представим $n$ в виде

$$
n=n_{1} n_{2}, \quad p\left(n_{1}\right) \leqslant z_{3}, \quad q\left(n_{2}\right)>z_{3} .
$$

Разобьем $X^{*}(N)$ на четыре суммы

$$
X^{*}(N) \leqslant X_{1}(N)+X_{2}(N)+X_{3}(N)+X_{4}(N) .
$$

В первой сумме $n=n_{1} n_{2}<N, n_{1} \leqslant z_{1}$, во второй $n=n_{1} n_{2}<N, n_{1} \geqslant z_{4}$, в третьей $n=n_{1} n_{2}<N, n_{1} \leqslant z_{4}, n_{2} \leqslant z_{2}$ и в четвертой $n=n_{1} n_{2}<N, z_{1}<n_{1} \leqslant z_{4}$, $z_{2}<n_{2},(n, N)=1, g(n) \in[a, a+1)$. Возьмем $z_{1}=\log ^{11} N, z_{3}=\exp \left((\log \log N)^{5}\right)$, $z_{4}=\exp \left(12(\log \log N)^{6}\right), z_{2}=N z_{4}^{-1} \log ^{-10} N$. Первая сумма будет оцениваться так:

$$
\leqslant 2 \sum_{n_{1} \leqslant z_{1}} \sum_{\substack{d \leqslant \sqrt{N} \\(d, N)=1}} \sum_{\substack{n_{2} \leqslant N / n_{1} \\ n_{1} n_{2} \equiv N(\bmod d)}} 1 .
$$


Так как $z_{3} \ll \sqrt[3]{N /\left(z_{1} d\right)}$, то для оценки суммы по $n_{2}$ можно применить решето Сельберга (см. [12, теорема 4.10]). Получим

$$
X_{1}(N) \ll \frac{N}{\log z_{3}} \sum_{n_{1} \leqslant z_{1}} \frac{1}{n_{1}} \sum_{d \leqslant \sqrt{N}} \frac{1}{\varphi(d)} \ll \frac{N \log N}{(\log \log N)^{4}} .
$$

Для оценки второй суммы воспользуемся неравенством Коши и леммой 1. Находим

$$
\begin{aligned}
X_{2}(N) & \leqslant\left(\sum_{n_{2} \leqslant N / z_{4}} \sum_{z_{4}<n_{1} \leqslant N / n_{2}} 1\right)^{1 / 2}\left(\sum_{n<N} \tau^{2}(n)\right)^{1 / 2} \\
& \ll N \exp \left(-\frac{\log z_{4}}{4 \log z_{3}}\right) \log ^{2} N \ll N \log ^{-1} N .
\end{aligned}
$$

Третья сумма оценивается так:

$$
\begin{aligned}
X_{3}(N) & \ll\left|\left\{n=n_{1} n_{2}: n_{1} \leqslant z_{4}, n_{2} \leqslant z_{2}\right\}\right|^{1 / 2}\left(N \log ^{3} N\right)^{1 / 2} \\
& \ll \sqrt{z_{4} z_{2} N} \log ^{3 / 2} N \ll N \log ^{-1} N .
\end{aligned}
$$

Возвращаясь к неравенству (11), получим

$$
\begin{aligned}
X^{*}(N) \leqslant & 2 \sum_{d \leqslant \sqrt{N}} \mid\left\{n=n_{1} n_{2}: z_{1}<n_{1} \leqslant z_{4}, z_{2}<n_{2} \leqslant \frac{N}{n_{1}}\right. \\
& g(n) \in(a, a+1], n \equiv N(\bmod d)\} \mid+O\left(\frac{N \log N}{(\log \log N)^{4}}\right) .
\end{aligned}
$$

Воспользуемся равенством

$$
\int_{-1}^{1}(1-|t|) e^{i t u} d u=\left(\frac{\sin (u / 2)}{u / 2}\right)^{2} .
$$

При $|u| \leqslant 1$ справедливы неравенства

$$
\frac{4}{\pi^{2}} \leqslant\left(\frac{\sin (u / 2)}{u / 2}\right)^{2} \leqslant 1
$$

Поэтому, оценив тривиально часть суммы по $d, Q \leqslant d \leqslant \sqrt{N}, Q=\sqrt{N} / \log N$, получим

$$
\begin{aligned}
& X^{*}(N) \leqslant 6 \sum_{d_{1} \leqslant Q} \sum_{\substack{d_{2} \leqslant Q / d_{1} \\
\left(d_{2}, N\right)=1}} \frac{1}{\varphi(d)} \sum_{\substack{n=n_{1} n_{2}<N \\
\left(n, N d_{2}\right)=1 \\
n \equiv N\left(\bmod d_{1}\right)}} \int_{-1}^{1}(1-|t|) e^{-i t(a-g(n))} d t \\
& +6 \sum_{\substack{d=d_{1} d_{2} \leqslant Q \\
\left(d_{1} d_{2}, N\right)=1}} \int_{-1}^{1}(1-|t|) \mid \sum_{\substack{z_{1}<n_{1} \leqslant z_{3} \\
\left(n_{1}, N d\right)=1}} e^{i t g\left(n_{1}\right)}\left(\sum_{\substack{z_{2}<n_{2} \leqslant N / n_{1} \\
\left(n_{1} n_{2} \equiv N(\bmod d)\right.}} e^{i t g\left(n_{2}\right)}\right. \\
& \left.-\frac{1}{\varphi\left(d_{2}\right)} \sum_{z_{2}<n_{2} \leqslant N / n_{1}} e^{i t g\left(n_{2}\right)}\right) \mid d t+O\left(\frac{N \log N}{(\log \log N)^{4}}\right) \text {. }
\end{aligned}
$$


В первой сумме мы снова вернулись к сумме по всем $n$, оценив, как и ранее, дополнительные суммы. Здесь $d=d_{1} d_{2}, p\left(d_{1}\right) \leqslant z, p\left(d_{2}\right)>z, z=(\log \log N)^{10}$. Применим лемму 6 , положив в ней $A=11$, а $z_{1}, z_{2}, z$ - теми же, что и выше. Получим, что вторая сумма в правой части оценивается так:

$\ll N \log ^{3 / 2} N(\log N)^{-11 / 2}$

$$
+\log \log N\left(\frac{\sqrt{N}}{\log N} \sqrt{N} \log \frac{N}{z_{1} z_{2}}+\left(\frac{N}{\sqrt{z_{2}}}+\frac{N}{\sqrt{z_{1}}}\right) \log ^{2} N+\frac{N}{z}(\log N) \log \frac{N}{z_{1} z_{2}}\right)
$$

$\ll \frac{N \log N}{(\log \log N)^{3}}$.

Заметим, что

$$
\sum_{\substack{d_{2} \leqslant Q \\\left(d_{2}, n N\right)=1}} \frac{1}{\varphi\left(d_{2}\right)} \leqslant \prod_{\substack{z<p \leqslant N \\ p \nmid N}}\left(1+\frac{p}{(p-1)^{2}}\right) \ll \prod_{\substack{p \mid N \\ p>z}} \frac{p-1}{p} \frac{\log N}{\log z}
$$

Таким образом, имеем

$$
\begin{aligned}
X^{*}(N) \ll & \frac{\log N}{\log z} \prod_{\substack{p \mid N \\
p>z}} \frac{p-1}{p} \sum_{\substack{d_{1} \leqslant Q \\
\left(d_{1}, N\right)=1}} \sum_{\substack{n<N \\
(n, N)=1 \\
n \equiv N\left(\bmod d_{1}\right)}} \int_{-1}^{1}(1-|t|) e^{-i t a} e^{i t g(n)} d t \\
& +N(\log N)(\log \log N)^{-3} .
\end{aligned}
$$

Часть первой суммы, в которой

$$
d_{1}>Q_{1}, \quad Q_{1}=\exp (6(\log z) \log \log \log N)=\exp \left(60(\log \log \log N)^{2}\right),
$$

будет оцениваться через

$$
\ll N \sum_{Q_{1}<d_{1} \leqslant Q} \frac{1}{d_{1}} \ll N \sum_{0 \leqslant k \leqslant \log \left(Q / Q_{1}\right)} \frac{1}{2^{k} Q_{1}}\left|\left\{d_{1} \leqslant 2^{k+1} Q_{1}, p\left(d_{1}\right) \leqslant z\right\}\right| .
$$

Применив лемму 1, получим, что эта величина оценивается так:

$$
\ll N \sum_{k \geqslant 0} \exp \left(-\frac{\log 2^{k+1} Q}{2 \log z}\right) \ll N(\log z)(\log \log N)^{-3}
$$

Следовательно,

$$
X^{*}(N) \ll \frac{\log N}{\log z} \prod_{\substack{p \mid N \\ p>z}} \frac{p-1}{p} X_{1}^{*}(N)+N(\log N)(\log \log N)^{-3},
$$


где

$$
\begin{aligned}
X_{1}^{*}(N) & =\sum_{\substack{d_{1} \leqslant Q_{1} \\
\left(d_{1}, N\right)=1}} \sum_{\substack{n<N \\
(n, N)=1 \\
n \equiv N\left(\bmod d_{1}\right)}} \int_{-1}^{1}(1-|t|) e^{-i t a} e^{i t g(n)} d t \\
& =\int_{-1}^{1} \sum_{\substack{d_{1} \leqslant Q_{1} \\
\left(d_{1}, N\right)=1}} \frac{1}{\varphi\left(d_{1}\right)} \sum_{\chi_{d_{1}}} \bar{\chi}_{d_{1}}(N) \sum_{\substack{n<N \\
(n, N)=1}} e^{i t g(n)} \chi_{d_{1}}(n)(1-|t|) e^{-i t a} d t .
\end{aligned}
$$

Для доказательства неравенства (10) нам достаточно показать, что

$$
X_{1}^{*}(N) \ll(\log z) \frac{\varphi(N)}{N} \prod_{\substack{p \leqslant z \\ p \mid N}}\left(1-\frac{1}{p}\right) \frac{N}{W(N)}
$$

Разобьем отрезок $[-1,1]$ на два множества следующим образом. Для $t \in U_{1}$ найдутся $\lambda(t),|\lambda(t)| \leqslant Q_{2}=\exp \left((\log \log \log N)^{3}\right)$, и характер Дирихле $\chi_{d}, d=d(t) \leqslant Q_{1}$, $p(d) \leqslant z$, такие, что

$$
\sum_{p<N} \frac{1}{p}\left(1-\operatorname{Re} \chi_{d}(p) e^{i t g(p)} p^{-i \lambda}\right) \leqslant(\log \log \log N)^{3}
$$

и $U_{2}=[-1,1] \backslash U_{1}$. Применяя лемму 2 и учитьвая, что

$$
\sum_{p \mid N} \frac{1}{p} \leqslant \log \log \log N+O(1)
$$

получим

$$
\begin{gathered}
\int_{U_{2}} \sum_{\substack{d_{1} \leqslant Q_{1} \\
\left(d_{1}, N\right)=1}} \frac{1}{\varphi\left(d_{1}\right)} \sum_{\chi_{d_{1}}} \bar{\chi}_{d_{1}}(N) \sum_{\substack{n<N \\
(n, N)=1}} e^{i t g(n)} \chi_{d_{1}}(n)(1-|t|) e^{-i t a} d t \\
\ll Q_{1} N \exp \left(-\frac{1}{3}(\log \log \log N)^{3}\right) \ll \frac{N}{(\log \log N)^{3}} .
\end{gathered}
$$

Пусть $t \in U_{1}$. Тогда найдутся $d=d(t) \leqslant Q_{1}, \lambda=\lambda(t) \leqslant Q_{2}$ такие, что имеет место неравенство (14), и поэтому

$$
\sum_{p<N} \frac{1}{p}\left|1-\chi_{d}(p) e^{i t g(p)} p^{-i \lambda}\right| \leqslant 2 \sqrt{\log \log N}(\log \log \log N)^{3 / 2} .
$$

Имеем

$$
\begin{aligned}
\sum_{\substack{n<N \\
(n, N)=1}} e^{i t g(n)} \chi_{d}(n) n^{-i \lambda} e^{i \lambda \log n}= & \sum_{\substack{n<N \\
(n, N)=1}} e^{i t g(n)} \chi_{d}(n) n^{-i \lambda} e^{i \lambda \log N} \\
& -i \lambda \int_{1}^{N} \sum_{\substack{n<u \\
(n, N)=1}} e^{i t g(n)} \chi_{d}(n) n^{-i \lambda} e^{i \lambda \log u} \frac{d u}{u} .
\end{aligned}
$$


Поэтому, применив лемму 4 , получим

$$
\begin{aligned}
& \sum_{\substack{n<N \\
(n, N)=1}} e^{i t g(n)} \chi_{d}(n)-\frac{\varphi(N)}{N} \sum_{n<N} e^{i t g(n)} \chi_{d}(n) \\
& \ll \sqrt{\sum_{d \mid N} \frac{1+\log d}{d} \frac{1}{\sqrt[4]{\log N}} \exp \left(\sqrt{\log \log N}(\log \log \log N)^{3 / 2}\right)} \\
& +\sqrt{\sum_{d \mid N} \frac{1+\log d}{d}} \exp \left(\sqrt{\log \log N}(\log \log \log N)^{3 / 2}\right) \int_{1}^{N} \frac{d u}{\sqrt[4]{\log u}} \\
& \ll \frac{N}{\sqrt[5]{\log N}} .
\end{aligned}
$$

Здесь мы воспользовались оценкой

$$
\sum_{d \mid N} \frac{1+\log d}{d} \ll \sum_{p^{r} \mid N} \frac{\log p^{r}}{p^{r}} \prod_{p \mid N}\left(1+\frac{1}{p}\right) \ll(\log \log N)^{2} .
$$

Таким образом, в интеграле по множеству $U_{1}$ в сумме по $n<N$ условие $(n, N)=1$ можно отбросить, поставив перед суммой множитель $\varphi(N) / N$. Возникающая при этом ошибка равна $O\left(Q_{1} N(\log N)^{-1 / 5}\right)=O\left(N \log ^{-1 / 6} N\right)$, т.е.

$$
\begin{aligned}
X_{1}^{*}(N)= & \frac{\varphi(N)}{N} \sum_{\substack{d_{1} \leqslant Q_{1} \\
\left(d_{1}, N\right)=1}} \frac{1}{\varphi\left(d_{1}\right)} \int_{U_{1}} \sum_{\chi_{d_{1}}} \bar{\chi}_{d_{1}}(N) \sum_{n<N} e^{i t g(n)} \chi_{d_{1}}(n)(1-|t|) e^{-i t a} d t \\
& +O\left(N(\log \log N)^{-3}\right) .
\end{aligned}
$$

Пусть $\chi_{\delta}^{*}-$ примитивный характер, порождающий характер $\chi_{d}$, с которым вьполнено неравенство (14). Тогда

$$
\sum_{p<N} \frac{1}{p}\left(1-\operatorname{Re} \chi_{\delta}^{*}(p) f(p) p^{-i \lambda}\right) \leqslant(\log \log \log N)^{3}+O(\log \log z) .
$$

Поэтому из леммы 3 для $t \in U_{1}$ следует, что для всех $\chi_{d_{1}}, d_{1} \leqslant Q, p\left(d_{1}\right) \leqslant z$, не порожденных $\chi_{\delta}$, при $|\lambda| \leqslant Q_{2}$ будет справедливо неравенство

$$
\sum_{p<N} \frac{1}{p}\left(1-\operatorname{Re} e^{-i t g(p)} \chi_{d_{1}}(p) p^{-i \lambda}\right) \geqslant \frac{1}{2} \log \log N+O(1) .
$$

Применив лемму 2 , получим, что в сумме по $\chi_{d_{1}}$ для каждого $t \in U_{1}$ можно оставить только слагаемые с $\chi_{d_{1}}$, где $\chi_{d_{1}}$ порожден $\chi_{\delta}^{*}$. Таким образом,

$$
\begin{aligned}
X_{1}^{*}(N) & \ll \frac{\varphi(N)}{N} \sum_{\substack{p\left(d_{1}\right) \leqslant z \\
\left(d_{1}, N\right)=1}} \frac{1}{\varphi\left(d_{1}\right)} N \int_{-1}^{1} \exp \left(-\frac{1}{8} M(t)\right) d t+O\left(N(\log \log N)^{-3}\right) \\
& \ll N \frac{\varphi(N)}{N} \prod_{\substack{p \leqslant z \\
p \mid N}}\left(1-\frac{1}{p}\right) \log z \int_{-1}^{1} \exp \left(-\frac{1}{8} M(t)\right) d t+O\left(N(\log \log N)^{-3}\right),
\end{aligned}
$$


где

$$
M(t)=\min _{\chi_{d}, \lambda} \sum_{p<N} \frac{1}{p}\left(1-\operatorname{Re} e^{-i t g(p)} \chi_{d}(p) p^{-i \lambda}\right) ;
$$

здесь минимум берется по всем $\lambda,|\lambda| \leqslant Q_{2}, \chi_{d}, d \leqslant Q_{1}, p(d) \leqslant z$. Подставляя в $(12)$, получим

$$
X_{1}^{*}(N) \ll\left(\frac{\varphi(N)}{N}\right)^{2} N \log N \int_{-1}^{1} \exp \left(-\frac{1}{8} M(t)\right) d t+O\left(N \log N(\log \log N)^{-3}\right) .
$$

\section{Положим}

$$
E_{k}=\{t: t \in[-1,1], M(t) \leqslant k\}, \quad k=1,2, \ldots,[9(\log \log \log N)]=K
$$

Тогда

$$
\int_{-1}^{1} \exp \left(-\frac{1}{8} M(t)\right) d t \ll \sum_{1 \leqslant k \leqslant K} \exp \left(-\frac{1}{8} k\right) m\left(E_{k}\right)+(\log \log N)^{-1} .
$$

Предположим, что для всех $1 \leqslant k \leqslant K$ вьполняется неравенство

$$
m\left(E_{k}\right) \leqslant \frac{200 k}{\sqrt{\log \log N}}
$$

Тогда мы получим, что оцениваемьй интеграл равен $O\left((\log \log N)^{-1 / 2}\right)$, и поэтому

$$
X_{1}^{*}(N) \ll\left(\frac{\varphi(N)}{N}\right)^{2} \frac{N \log N}{\sqrt{\log \log N}} \ll\left(\frac{\varphi(N)}{N}\right)^{2} \frac{N \log N}{\sqrt{W(N)}} .
$$

То есть мы получим утверждение теоремы 1.

Предположим, что найдется $1 \leqslant k \leqslant K$, для которого

$$
m\left(E_{k}\right)>\frac{200 k}{\sqrt{\log \log N}} .
$$

Множество $E_{k}$ симметрично относительно нуля и содержит нуль, поэтому (см., например, $\left[1\right.$, лемма 5.3]) каждое число $t \in[-1,1]$ представимо в виде $t_{1}+t_{2}+\cdots+t_{r}$, где $t_{j} \in E_{k}$ и $r=\left[12 / m\left(E_{k}\right)\right]$. Напомним, что для каждого $t_{j} \in E_{k}$ найдутся характер $\chi_{d_{j}}, d_{j} \leqslant Q_{1}, p\left(d_{j}\right) \leqslant z$, и число $\lambda_{j}=\lambda\left(t_{j}\right),\left|\lambda\left(t_{j}\right)\right| \leqslant Q_{2}$, такие, что

$$
\sum_{p<N} \frac{1}{p}\left(1-\operatorname{Re} e^{i t_{j} g(p)} p^{-i \lambda_{j}} \chi_{\delta_{j}}(p)\right) \leqslant k .
$$

Заметим, что при любом натуральном $r$ и любых комплексных числах $z_{j},\left|z_{j}\right| \leqslant 1$, справедливы неравенства

$$
\begin{aligned}
1- & \operatorname{Re}\left(z_{1} \cdots z_{r}\right) \\
& \leqslant 1-\operatorname{Re}\left(z_{1} \cdots z_{r-1}\right)+\left|\operatorname{Im}\left(\operatorname{Re}\left(z_{1} \cdots z_{r-1}\right)\right)\right| \cdot\left|\operatorname{Im} z_{r}\right|+\operatorname{Re}\left(z_{1} \cdots z_{r-1}\right)\left(1-\operatorname{Re} z_{r}\right) \\
& \leqslant 1-\operatorname{Re}\left(z_{1} \cdots z_{r-1}\right)+2 \sqrt{\left(1-\operatorname{Re}\left(z_{1} \cdots z_{r-1}\right)\right)} \sqrt{1-\operatorname{Re} z_{r}}+\left(1-\operatorname{Re} z_{r}\right) .
\end{aligned}
$$


Следовательно, по индукции получим

$$
\begin{aligned}
1-\operatorname{Re}\left(z_{1} \cdots z_{r}\right) \leqslant & (r-1)^{2} \sum_{j=1}^{r-1}\left(1-\operatorname{Re} z_{j}\right) \\
& +2(r-1) \sqrt{\sum_{j=1}^{r-1}\left(1-\operatorname{Re} z_{j}\right)\left(1-\operatorname{Re} z_{r}\right)}+\left(1-\operatorname{Re} z_{r}\right) \\
\leqslant & r^{2} \sum_{j=1}^{r}\left(1-\operatorname{Re} z_{j}\right) .
\end{aligned}
$$

Таким образом, для любого $t \in[-1,1]$ мы имеем $t=t_{1}+\cdots+t_{r}$, где $t_{j} \in E_{k}$, и поэтому при $\lambda=\lambda(t)=\lambda_{1}+\cdots+\lambda_{r}, \chi_{d}=\chi_{d_{1}} \cdots \chi_{d_{r}}, d=d(t)=\left[d_{1}, \ldots, d_{r}\right]$, получим

$$
\sum_{p<N} \frac{1}{p}\left(1-\operatorname{Re} e^{i t g(p)} p^{-i \lambda} \chi_{d}(p)\right) \leqslant r^{2} k \leqslant 13^{2} \frac{\log \log N}{200^{2} k} \leqslant \frac{\log \log N}{102} .
$$

Заметим, что $|\lambda| \leqslant r Q_{2} \leqslant Q_{2} \sqrt{\log \log N}, d=d(t) \leqslant Q_{1}^{r} \leqslant \log N, p(d) \leqslant z$. Покажем, что $\chi_{d}$ - главный характер. Рассмотрим любые $t_{1}, t_{2} \in[-1,1]$ такие, что $t_{1}+t_{2} \in[-1,1]$. Покажем, что характер $\chi_{\Delta}=\chi_{d\left(t_{1}+t_{2}\right)} \bar{\chi}_{d\left(t_{1}\right)} \bar{\chi}_{d\left(t_{2}\right)}$ главнњй. Используя неравенства (18) и (17), получим

$$
\sum_{p<N} \frac{1}{p}\left(1-\operatorname{Re} \chi_{\Delta}(p) p^{-i\left(\lambda\left(t_{1}+t_{2}\right)-\lambda\left(t_{1}\right)-\lambda\left(t_{2}\right)\right.}\right) \leqslant 9 \frac{\log \log N}{100} \leqslant \frac{\log \log N}{10}
$$

Положим $\sigma_{N}=1+1 / \log N$. Тогда левая часть неравенства равна

$$
\log \zeta\left(\sigma_{N}\right)-\log \left|L\left(\sigma_{N}+i\left(\lambda\left(t_{1}\right)+t_{2}\right)-\lambda\left(t_{1}\right)-\lambda\left(t_{2}\right)\right), \chi_{\Delta}\right|+O(1)
$$

Если $\chi_{\Delta}-$ неглавный характер, то

$$
\zeta\left(\sigma_{N}\right)=\log N+O(1), \quad L\left(\sigma_{N}+i t, \chi_{\Delta}\right)=O(\ln |\Delta|(|t|+2))
$$

т.е. получим противоречие. Таким образом, $\chi_{\Delta}$ - главньй характер. Положим

$$
H=\left(\prod_{p \leqslant z} p\right)^{s}, \quad \text { где } s=\left[\log Q_{1}\right][2 \sqrt{\log \log N}] .
$$

Тогда модули всех характеров, о которых шла речь вьше, будут делить $H$. Будем обозначать через $\tilde{\chi}_{d}$ характер по модулю $H$, порожденный $\chi_{d}$. Тогда для любых $t_{1}, t_{2} \in$ $[-1,1], t_{1}+t_{2} \in[-1,1]$, имеем $\widetilde{\chi}_{d\left(t_{1}+t_{2}\right)}=\tilde{\chi}_{d\left(t_{1}\right)} \tilde{\chi}_{d\left(t_{2}\right)}$, и поэтому при $h=\varphi(H)$ получим $\tilde{\chi}_{d(t)}=\left(\tilde{\chi}_{d(t / h)}\right)^{h}=\tilde{\chi}_{0}$, где $\widetilde{\chi}_{0}-$ главный характер модуля $H$. То есть мы доказали, что $\chi_{d(t)}-$ главньй характер. Учитьвая, что

$$
\sum_{p \mid d} \frac{1}{p} \ll \log \log z
$$


и неравенство (18), мы получим, что для любого $t \in[-1,1]$ существует $\lambda=\lambda(t)$, $|\lambda(t)| \leqslant \log N$, такое, что

$$
\sum_{p<N} \frac{1}{p}\left(1-\operatorname{Re} e^{i t g(p)} p^{-i \lambda}\right) \leqslant \frac{\log \log N}{100} .
$$

Снова применив леммы 3 и 2 и воспользовавшись (15), получим

$$
\begin{aligned}
X_{1}^{*}(N) & \ll \frac{\varphi(N)}{N} \sum_{n \leqslant N}\left(\frac{\sin ((g(n)-a) / 2)}{(g(n)-a) / 2}\right)^{2} \sum_{\left(d_{1}, N\right)=1} \frac{1}{\varphi\left(d_{1}\right)}+\frac{N}{(\log \log N)^{3}} \\
& \ll \frac{\varphi(N)}{N} \prod_{\substack{p \leqslant z \\
p \mid N}}\left(1-\frac{1}{p}\right) \log z \sum_{n \leqslant N}\left(\frac{\sin ((g(n)-a) / 2)}{(g(n)-a) / 2}\right)^{2}+\frac{N}{(\log \log N)^{3}} .
\end{aligned}
$$

Отсюда и из (12) следует оценка

$$
X^{*}(N) \ll\left(\frac{\varphi(N)}{N}\right)^{2} \log N \sup _{a}|\{n: n \leqslant N, g(n) \in[a, a+1]\}|+\frac{N}{(\log \log N)^{2}} .
$$

Применив результат Ружи (см. [1]), мы завершим доказательство теоремы 1.

Авторы благодарят рецензента за полезные замечания.

\section{СПИСОК ЦИТИРОВАННОЙ ЛИТЕРАТУРЫ}

[1] Ruzsa I. On the concentration of additive functions // Acta Math. Acad. Sci. Hungar. 1980. V. 36. P. 215-232.

[2] Halasz G. On the distribution of additive arithmetic functions // Acta Arith. 1974. V. 27. P. 143-152.

[3] Тимофеев Н. М. Гипотеза Эрдеша-Кубилюса о распределении значений аддитивных функций на последовательности сдвинутых простых чисел // Acta Arith. 1991. V. 58. № 2. P. 113-131.

[4] Elliott P.D.T.A. The concentration function of additive functions on shifted primes // Acta Math. 1994. V. 173. P. 1-35.

[5] Тимофеев Н. М., Хрипунова М. Б. Распределение чисел с заданньм числом простых делителей в прогрессиях // Матем. заметки. 1994. Т. 55. № 2. С. 144-156.

[6] Тимофеев Н. М., Хрипунова М. Б. Проблема Титчмарша с числами, имеющими заданное число простых делителей // Матем. заметки. 1996. Т. 59. №4. С. 586-603.

[7] Tenenbaum G. Introduction à la theorie analytique et probabiliste des nombres: Institut E. Cartan, 13. Université de Nancy I, 1990.

[8] Halasz G. Über die Mittelwerte multiplikativer zahlentheoretischer Funktionen // Acta Math. Acad. Sci. Hungar. 1968. V. 19. P. 365-403.

[9] Elliott P. D. T. A. Probabilistic Number Theory I. Mean Value Theorems. Grundlehren Math. Wiss. V. 239. New York-Heidelberg-Berlin: Springer Verlag, 1979.

[10] Левин В. В., Тимофеев Н. М. Распределение арифметических функций в среднем попрогрессиям (теоремы типа Виноградова-Бомбьери) // Матем. сб. 1984. Т. 125. №4. С. 558-572.

[11] Монтгомери Г. Мультипликативная теория чисел. М.: Мир, 1974.

[12] Прахар К. Распределение простых чисел. М.: Мир, 1967.

Владимирский государственный педагогический университет

Поступило

E-mail: hripunova@vgpu.vladimir.ru

16.08 .2000

Исправленный вариант

10.11 .2002 